\title{
Contribuciones de la Unión Europea a los desafíos que representa la protección del medio ambiente: La diplomacia europea en materia de lucha contra el cambio climático*,1
}

\author{
Contributions of the European Union to Environmental Protection \\ Challenges: The European Diplomacy Towards Climate Change
}

\author{
Justo Corti Varela \\ Profesor Adjunto de Derecho Internacional y Europeo \\ Instituto de Estudios Europeos \\ Universidad CEU San Pablo \\ jlcorti@ceu.es
}

doi: http://dx.doi.org/10.18543/ced-57-2017pp167-192

\begin{abstract}
Sumario: I. Introducción.-II. Las razones internas. 1. ¿Por qué los Estados Unidos cedieron a la UE el liderazgo en materia de cambio climático? 2. La diplomacia interna de la UE y el problema de la competitividad.-III. La evolución del liderazgo europeo. 1. De la Convención sobre el Cambio Climático al Protocolo de Kioto. 2. El fracaso de Copenhague. 3. El renacer de la UE como «líder-mediador»: de Durban a Paris. - IV. El liderazgo europeo más allá de las instituciones de la UE: El rol jugado por la presidencia francesa en los acuerdos de París.-V. Conclusiones.-VI. Bibliografía.
\end{abstract}

Resumen: En la literatura sobre cambio climático existe un consenso en que la UE (Unión Europea) ha sido la gran impulsora de acuerdos, tanto por su rol activo en las negociaciones como su labor en el diseño de un modelo de economía sostenible. Sin embargo, no ha sido hasta el «fracaso» de Copenhague en que se haya estudiado con más profundidad el mecanismo de actuación de la diplomacia europea en la materia, las estrategias seguidas, las razones de los éxitos y los fracasos. En este trabajo analizaremos, en primer lugar, cómo la UE se convirtió en

* Recibido el 12 de enero de 2017 y aceptado el 30 de abril de 2017.

${ }^{1}$ El presente estudio se enmarca dentro del proyecto de investigación: «Las Respuestas del Derecho Internacional y Europeo a los Nuevos Riesgos y Amenazas Contra la Seguridad Humana» (RASEGUR), Plan Nacional de I+D+I (Ref.: DER2015-65906-P) y de la Red de Excelencia sobre «Los actuales desafíos del Derecho Internacional», del Plan Estatal de Investigación Científica y Técnica y de Innovación 2013-2016 (DER15-69273-RED). Un agradecimiento especial a los pares ciegos por sus comentarios y sugerencias así como a la Prof. Rosa Giles Carnero por las ideas vertidas en las etapas iniciales de esta investigación. 
el líder en materia de acuerdos sobre cambio climático, los necesarios consensos internos a que se tuvo que arribar para tener una postura aceptable para todos y la evolución de las diferentes estrategias seguidas, desde los éxitos de Kioto (1997) y Paris (2015), pasando por el fracaso de Copenhague (2009) y el cambio iniciado en Durban (2011).

Palabras clave: diplomacia europea - cambio climático - «líder/mediador» Acuerdo de Paris

Abstract: In the literature on climate change there is an extended consensus about the EU (European Union) leadership in the agreement making, including its active role in negotiation and its contributions in the design of a model of sustainable economy. However, it was not until the failure of Copenhagen when scholars studied more in detail how the European diplomacy acts in practice, the different strategies followed and the reasons for success and breakdown. In this article we will analyze, firstly, how the EU has built its leadership in the promotion of climate change agreements, the necessary internal consensus that it had to achieve in order to present a common position and the evolution of different strategies, from the success of Kyoto (1997) and Paris (2015), to the failure of Copenhagen (2009) and the big change initiated in Durban (2011).

Keywords: European diplomacy - climate change - «leadiator» - Paris agreement

\section{Introducción}

Si bien hoy en día nadie discute que la UE tiene un rol destacado en la promoción y firma de acuerdos internacionales tendientes a la protección del medio ambiente, y que en dichos foros se enfrenta a visiones diferentes defendidas por otros socios como Estados Unidos o más recientemente China, esto no siempre fue así. En los años 70 y 80 del siglo pasado el liderazgo en materia lo ostentaba los Estados Unidos y la Comunidad Europea (CE), o los países que en ese momento la formaban y ejercían plenamente las competencias internacionales en la materia, tenían una posición más bien pasiva, de acompañamiento al liderazgo americano. ${ }^{2}$

${ }^{2}$ R. Daniel Kelemen y Tim Knievel, «The United States, the European Union, and international environmental law: the domestic dimensions of green diplomacy!», International Journal of Constitutional Law 13, n. ${ }^{\circ} 4$ (2015): 946. Para una evolución histórica sobre el liderazgo europeo en la materia ver Rüdiger K.W. Wurzel, Duncan Liefferink y James Connelly «Introduction. European Union climate leadership» en The European Union in International Climate Change Politics: Still Taking a Lead?, ed. por Rudiger K.W. Wurzel, James Connelly y Duncan Liefferink (Abingdon: Routledge, 2017): 1-17. 
A partir de 1990 estos roles se intercambiaron ${ }^{3}$ pero a diferencia del período anterior en la nueva etapa los Estados Unidos no solo no lideraron la promoción ni la firma de acuerdos medioambientales sino que en muchos casos boicotearon las iniciativas europeas. En algunos supuestos simplemente no ratificaron los acuerdos ${ }^{4}$, en otros rechazaron su firma ${ }^{5}$ e incluso en algunos, como ocurrió en Copenhague, hicieron todo lo posible para decretar su fracaso ${ }^{6}$. Resulta interesante estudiar las causas de este cambio, como haremos en el apartado II de este artículo, ya que, en primer lugar, la UE puede llegar a sufrir un proceso similar si llegasen a repetirse esta vez en territorio europeo las condiciones internas que generaron la desafección americana al cambio climático y; en segundo término y tal como enseña la experiencia del Protocolo de Kioto, porque la UE está condenada a entenderse con su socio trasatlántico (y también con China) si quiere influir realmente en la agenda medioambiental global. Uno de los puntos de contacto es, especialmente, el problema de la pérdida de competitividad que genera una agenda que no incluya a todos los actores principales del cambio climático. El llamado «carbon leakage» ha sido esencial en el proceso de consenso interno europeo y, seguramente, pasará a ser muy importante en las negociaciones post-París ya que al ser un esquema flexible cada Estado intentará concentrar su margen de maniobra en aquellas actividades más vulnerables desde el punto de vista del comercio internacional.

El apartado III estudia la evolución histórica del liderazgo europeo en las negociaciones climáticas. Desmitificando algunos elementos del relato europeo, esencial en los noventa para justificar la creación de una política

${ }^{3}$ R. Daniel Kelemen y David Vogel, «Trading places: the role of the United States and the European Union in international environmental politics», Comparative Political Studies 43, n. ${ }^{\circ} 4$ (2010): 427-456.

${ }^{4}$ Los Estados Unidos no ratificaron la Convención sobre la Diversidad Biológica (1992), la Convención de Helsinki sobre los efectos transfronterizos de los accidentes industriales (1992), el Protocolo de Kyoto (1997), el Convenio de Róterdam sobre el procedimiento de consentimiento fundamentado previo aplicable a ciertos plaguicidas y productos químicos peligrosos objeto de comercio internacional (1998), el Convenio de Estocolmo sobre los Contaminantes Orgánicos Persistentes (2001) o el Tratado Internacional sobre los Recursos Fitogenéticos para la Alimentación y la Agricultura (2001).

5 Por ejemplo la Convenio sobre acceso a la información, participación del público en la toma de decisiones y acceso a la justicia en materia de medio ambiente (1998) o el Protocolo de Cartagena sobre Seguridad de la Biotecnología (2000).

${ }^{6}$ Es conocida la anécdota de que el tibio acuerdo final alcanzado en Copenhague se tomó en un momento en que el negociador europeo no estaba presente en la mesa. Charles F. Parker, Christer Karlsson y Mattias Hjerpe «Assessing the European Union's global climate change leadership: from Copenhagen to the Paris Agreement» Journal of European Integration 39, n. 2 (2017): 239-252, DOI: 10.1080/07036337.2016.1275608. 
exterior y paliar, en parte, los fracasos sonoros de la guerra de Yugoslavia y de Irak, analizamos la actuación de la UE primero en Kioto y luego en Copenhague. Si bien la estrategia fue parecida, montada sobre la idea de un liderazgo duro con muy poca negociación, las circunstancias históricas llevaron al primero a ser un éxito relativo (teniendo en cuenta el número real de Estados vinculados) y al segundo un fracaso rotundo. El gran cambio hacia un liderazgo «mediador» llegará con Durban y recogerá sus frutos en Paris. La pregunta que nos hacemos, y trataremos de responder en las conclusiones, es hasta qué punto París no ha sido también, como Kioto, un producto de las circunstancias y si la nueva administración americana cumple sus promesas de desandar lo acordado por el presidente Obama no nos encontraremos nuevamente ante un escenario de desequilibrio en las obligaciones?

Finalmente, en el apartado IV, se analizará el papel jugado por la presidencia francesa en los acuerdos de París, en clave de liderazgo europeo general, no necesariamente de la UE. Teniendo en cuenta que la acción exterior de la UE es una política compleja, donde los Estados miembros tienen un rol crucial, se analizará hasta qué punto la actuación de un Estado en particular, en este caso Francia, puede ser considerado un complemento a la acción de la UE y, en su conjunto, parte de su estrategia de liderazgo en el ámbito del cambio climático.

\section{Las razones internas}

Todo liderazgo internacional se construye previamente en el fuero interno. Si no existe un consenso interno fuerte es muy difícil desarrollar una política exterior coherente. El liderazgo europeo en materia de cambio climático no es la excepción. Pero en este caso, dado lo reciente de las competencias exteriores europeas, cuando la UE apostó de lleno por dicho liderazgo debió compartir el mérito no sólo en las virtudes internas sino también en el retroceso voluntario que realizó el entonces líder (Estados Unidos). Por lo tanto, no estamos, en realidad, ante un enfrentamiento de liderazgos como el ocurrido en la guerra fría sino más bien al abandono voluntario de uno y la ocupación de ese espacio por otro. Comprender las razones internas de ambos cambios nos ayudan a entender no sólo los reflejos que luego tuvieron en la esfera internacional, sino también nos pueden dar pistas de los movimientos futuros de cada uno, incluidos los problemas a que se puede enfrentar la UE en el supuesto de que se den condiciones parecidas a las vividas en los Estados Unidos en el momento del intercambio de roles. 


\section{1. ¿Por qué los Estados Unidos cedieron a la UE el liderazgo en materia de cambio climático?}

Como explica con detalle Pamela Chasek, la principal causa del desinterés americano por los acuerdos medioambientales a partir de 1990 es, en realidad, fruto de una aversión hacia el multilateralismo en general y, en particular, a aquel vinculado con la protección de los derechos sociales y económicos ${ }^{7}$. Probablemente la industria americana fue la primera que comprendió que la lucha contra el cambio climático era en realidad una guerra económica y ellos no estaban dispuestos a comprometerse a nada que pudiese alterar su modelo de producción.

Sin embargo, si abandonamos un poco los estereotipos e intentamos ahondar en los focos de resistencia dentro del entramado institucional americano resulta interesante ver que el retroceso americano no siempre formó parte de la estrategia general de la Secretaría de Estado (diplomacia central) sino más bien fue consecuencia de una oposición contundente del Senado, órgano legislativo encargado de ratificar los tratados internacionales. En esta institución, donde están representados los intereses de los Estados frente al poder central de la presidencia, es particularmente renuente a ceder competencias sea a una organización internacional sea a una agencia federal. Además, es una institución particularmente conservadora ya que en ella tienen igual peso todos los Estados sin importar su población o economía, con un aclara sobre-representación de miembros del centro-oeste, interior y rural. Por ejemplo, el Senado, bajo el control del partido republicano, rechazó ratificar la Convención de Estocolmo sobre Contaminantes Orgánicos Persistentes a pesar que los contaminantes prohibidos por dicho acuerdo ya eran ilegales en los Estados Unidos desde hacía décadas ¿Por qué el rechazo entonces? Porque la convención podía ampliar en el futuro el número de prohibiciones y, de ser así, la autoridad nacional de ejecución (la Environmental Protection Agency) acrecentaría indirectamente sus competencias ${ }^{8}$.

En esta lucha interna y externa por mantener las competencias la fijación de los propios estándares parece ser un factor importante. Los Estados Unidos y la UE son los únicos dos actores con capacidad de imponer sus modelos a nivel internacional ${ }^{9}$. Sin embargo, la creación de los estándares propios

${ }^{7}$ Pamela Chasek «US policy in the UN environmental arena: powerful laggard or constructive leader?», International Environmental Agreements: Politics, Law and Economics 7, n. 4 (2007): 363-387.

8 Kelemen y Knievel (2015): 952.

9 Para ver las razones de esta afirmación ver Norman J. Vig y Michael Gebert Faure (eds.) Green Giants? Environmental Policies of the United States and the European Union (Cambridge: MIT Press, 2004), esp. la introducción de los editores. 
es un proceso interno que se desarrolla según parámetros locales. A partir de los años ochenta el movimiento por los derechos civiles en los Estados Unidos comenzó a perder fuerza y, en particular, dejó de centrarse en las causas vinculadas con el medioambiente. Por el contrario en dicha misma época los partidos verdes europeos comenzaron a consolidarse ${ }^{10}$ y a imponer su agenda. Esto explica en cierto modo por qué la legislación medioambiental americana dejó de evolucionar y la europea pasó a ocupar la delantera. La cuestión no es baladí ya que cuanto más estrictos sean los estándares internos, más cohesión habrá para tratar de reflejar esos mismos estándares a nivel internacional, promoviendo o apoyando acuerdos que los incluyan. Esto es así por tres razones, en primer lugar por simple coherencia; en segundo término, para evitar perder competitividad (tal como analizaremos en el siguiente punto); y en tercer lugar, para promover la propia industria medioambiental que creció al calor de la normativa interna y que al ver extender el marco normativo que hizo posible su modelo de negocio a otros países puede encontrar oportunidades de expansión hacia el extranjero ${ }^{11}$.

$\mathrm{El}$ freno de la normativa medioambiental americana, que en definitiva hará perder su liderazgo internacional, responde a razones ideológicas más que económicas. Este argumento es difícil de explicar ya que la concienciación ecologista no es necesariamente un elemento ideológico. Al menos así se entiende en Europa. De hecho, es normal que en los países como más tradición en la protección del medioambiente tanto partidos de derecha como de izquierda apoyen las medidas de protección. Sin embargo en los Estados Unidos, en especial a partir de la administración Reagan ${ }^{12}$, el partido republicano vinculó la protección del medioambiente en general y la del cambio climático en particular a un atentado contra la propiedad. El inicio del cambio fue el informe especial The Specter of Environmentalism (1982) redactado por la House Republican Study Committee que califica a los ambientalistas de «extremistas», una mezcla de liberales, revolucionarios y «contraculturales» que quieren contaminar los fundamentos sociales y políticos del país con una nueva ideología ${ }^{13}$. El discurso se mantuvo en

${ }^{10}$ Sobre el desarrollo del movimiento ambientalista en Europa y su transformación en partidos políticos ver Yves Frémion: Histoire de la révolution écologiste (Paris: Hoëbeke, 2007), esp. capítulo 6.

${ }^{11}$ Para un estudio de estas causas ver David Vogel, The politics of precaution: regulating health, safety, and environmental risks in Europe and the United States (Princeton: Princeton University Press, 2012): 235-248.

12 Antes no era así. Por ejemplo la Clean Air Act, pionera en la protección de la calidad del aire, fue promovida por el Presidente Nixon a inicios de los años 70.

13 Para un comentario de este informe y sus repercusiones en el conservadurismo americano ver: James Morton Turner, «The Specter of Environmentalism: Wilderness, Environmental Politics, and the Evolution of the New Right», The Journal of American History 96, n. ${ }^{\circ} 1$ (2009): 123-148. 
varios grupos de presión, como por ejemplo el Competitive Enterprise Institute que continuaron alimentando esta visión y pusieron en duda las pruebas científicas sobre el cambio climático ${ }^{14}$. El senador Malcom Wallop, del partido republicano, sintetizó este sentimiento al hablar de su rechazo a la Convención sobre la Diversidad Biológica:

«The administration has fought to subordinate private property rights to the newly proclaimed rights of various plant and animal species... The biodiversity treaty, as written, would give the Clinton administration even greater authority to accomplish suspect environmental goals.» ${ }^{15}$

Inclusive durante las presidencias demócratas de Clinton y Obama no se lograron grandes avances por el bloqueo republicano sea en el Congreso o en el Senado. Por ejemplo la American Clean Energy and Security Act (2009), que incluía un sistema de comercio de emisiones, no llegó a aprobarse por falta de acuerdo en la asignación de los derechos de emisión. En la reciente campaña presidencial lejos de cambiar la posición republicana, y finalmente vencedora, no hizo más que reforzarse. En las primarias del partido republicano todos los candidatos se manifestaron contrarios a adoptar medidas para revertir el cambio climático, llegando incluso varios a seguir negando su existencia ${ }^{16}$. La recientes declaraciones del presidente electo Donald Trump de revisar el presupuesto de la NASA destinado a la investigación sobre cambio climático por considerarlo «ciencia politizada» ${ }^{17}$, la posible salida de los acuerdos de Paris y la nominación dentro de su gabinete, de Rex Tillerson ${ }^{18}$ para el Departamento de Estado, Scott

${ }^{14}$ Para un análisis en detalle de la actuación de ese grupo de presión junto a otros ver Naomi Oreskes y Erik M. Conway, Merchants of doubt: How a handful of scientists obscured the truth on issues from tobacco smoke to global warming (Nueva York: Bloomsbury Publishing, 2011): 169-215 .

15140 Congress Records (30 septiembre 1994), acceso 30 de diciembre 2016, www.gpo. gov/fdsys/pkg/CREC-1994-09-30/html/CREC-1994-09-30-pt1-PgS22.htm.

16 Republican Views, «Republican Views on Global Warming», post publicado el 5 de marzo de 2016, acceso 30 de diciembre 2016, http://www.republicanviews.org/republicanviews-on-global-warming-2/.

17 The Guardian «Trump to scrap Nasa climate research in crackdown on "politicized science"» 22 de noviembre 2016, acceso 30 de diciembre 2016, https://www theguardian. com/environment/2016/nov/22/nasa-earth-donald-trump-eliminate-climate-change-research.

18 Tillerson, de Exxon Mobil, una compañía cuya posición oficial durante años ha sido la de negar la existencia del cambio climático. Se le atribuye la frase de que el cambio climático no es una cuestión realmente importante y, de existir, «ya nos adaptaremos a él». The Guardian «Climate change fears overblown, says ExxonMobil», 28 de junio de 2012, acceso 30 de diciembre 2016, fttps://www theguardian.com/environment/2012/jun/28/exxonmobil-climatechange-rex-tillerson. 
Pruitt ${ }^{19}$ para la Environmental Protection Agency o Rick Perry ${ }^{20}$ para el Departamento de Energia, seguramente dejará libre el camino al liderazgo europeo en la materia.

\section{La diplomacia interna de la UE y el problema de la competitividad}

Que la UE haya logrado mantener una política exterior en materia climática coherente a lo largo de los años no responde sólo al desinterés americano sino que engloba méritos propios. La política europea en materia climática viene precedida de un ejercicio de diplomacia interna para poner de acuerdo a 28 Estados que no tienen necesariamente la misma visión del problema, algo que no ha sido posible en otras esferas de la política exterior ${ }^{21}$.

Probablemente uno de los mayores logros de la política climática europea fue unirla a la política energética. Esto tiene claras ventajas en términos de coherencia pero presenta el problema competencial de que la política energética en general, y la construcción del mix energético en particular, están todavía muy poco comunitarizadas. Por ello no es extraño que existan diferentes visiones internas de cómo hacer frente al cambio climático cuando las medidas inciden en las fuentes de producción energética.

Hay por lo menos dos grandes visiones, la del grupo Visegrad, liderado por Polonia ${ }^{22}$, y la del grupo Crecimiento Verde (Green Growth) liderado especialmente por Alemania, Dinamarca y Francia ${ }^{23}$. Dentro de este último,

19 Pruitt es abogado general de Oklahoma y como tal ha encabezado varios pleitos contra la EPA para no aplicar en su Estado las regulaciones de dicha agencia para limitar las emisiones de las plantas generadoras de electricidad. Kate Sheppard «Donald Trump Picks Fossil Fuel-Friendly Oklahoma Attorney General To Head EPA» The Huffington Post, 12 julio 2016, acceso 30 de diciembre 2016, http://www.huffingtonpost.com/entry/trump-pruittenvironmental-protection-agency_us_584856f1e4b0b9feb0dab14f

${ }^{20}$ Perry, antiguo gobernador de Texas, dijo que el cambio climático es «una teoría que aun no ha sido demostrada» y que los científicos que lo estudian «manipulan los datos para mantener la financiación». Kate Sheppard «It's hard to overstate how anti-environment Donald Trump's Cabinet Picks Are» The Huffington Post, 13 diciembre 2016, acceso 30 de diciembre 2016, http://www.huffingtonpost.com/entry/trump-administrationanti-environment_us_58507c5ae4b0ee009eb44512.

${ }^{21}$ Lisanne Groen y Arne Niemann, «The European Union at the Copenhagen Climate Negotiations: A Case of Contested EU Actorness and Effectiveness» International Relations 27, n. 3 (2013): 308-324.

${ }^{22}$ Participan en él, además de Polonia, otros países del centro y este de Europa como Bulgaria, Republica Checa, Hungría, Rumania y Eslovaquia.

${ }^{23}$ España forma parte de este grupo y en él intervienen también Bélgica, Estonia, Finlandia, Italia, Holanda, Portugal, Eslovenia, Suecia y, con sus particularidades, Reino Unido. 
sin embargo, hay diferencias importantes en cuando a la transición energética. Alemania, por ejemplo, prefiere pasar directamente a las renovables mientras que Reino Unido, al menos antes del Brexit, opinaba que la energía nuclear y el gas eran pasos intermedios insalvables.

Las diferentes visiones coinciden, no obstante, en un tema esencial con claras repercusiones internacionales: cómo evitar que la política climática europea socave la competitividad internacional de nuestra economía.

Uno de los principales temores del sistema de derechos de emisión que se instauró en el Protocolo de Kioto fue que, debido a que muchos de los socios comerciales de la UE no eran parte del sistema, el progresivo aumento de los derechos incentivaran la deslozalización de las industrias más intensivas en el uso de energía ${ }^{24}$.

Si bien el «carbon leakage» ha sido objeto de estudio en diferentes trabajos ${ }^{25}$, todavía no ha sido posible demostrar una clara relación causaefecto $^{26}$ ya que el fenómeno de la deslocalización depende de diversos factores como la incidencia de los derechos de emisión en la estructura de costes o la capacidad que tiene el sector para trasladar dichos costes a otros sectores o a los propios consumidores. Este último elemento puede ser determinante y, en él, la estructura del mercado y en especial el adecuado funcionamiento de las reglas de la competencia ${ }^{27}$. La Comisión Europea ha visto riesgos en algunos sectores de alta intensidad energética (cemento, aluminio, acero, papel) aunque en dicho estudio no se considera la estructura del mercado ni la repercusión de los costes a los consumidores, con lo que los riesgos reales parecen ser bastante más reducidos. ${ }^{28}$

Para curarse en salud, y garantizar la necesaria cohesión interna de cara a las negociaciones de Copenhague, en 2009 la Comisión Europea decidió aumentar la asignación de derechos de emisión a todas las industrias con

${ }^{24}$ Madison Condon y Ada Ignaciuk «Border Carbon Adjustment and International Trade: A Literature Review» OECD Trade and Environment Working Papers, n. ${ }^{\circ} 6$ (2013).

${ }^{25}$ Para un resumen de la literatura ver Andrei Marcu, et al. «Carbon Leakage: An overview» CEPS Special Report (2013), acceso 30 de diciembre 2016, www.ceps.be/book/carbonleakage-overview.

${ }^{26}$ Ralf Martin, Laure de Preux y Ulrich Wagner «The impacts of a carbon tax on manufacturing: Evidence from microdata» Journal of Public Economics 117, n. ${ }^{\circ} 1$ (2014): 1-14.

27 Justo Corti Varela «EU Emission Trading System, competitiveness and taxes on the energy sector» en State Aids, Taxation and the Energy Sector, ed. por Marta Villar Ezcurra (Cizur Menor: Thomson Reuters, 2017), 323-336.

${ }_{28}$ Sander de Bruyn, Dagmar Nelissen y Marnix Koopman «Carbon leakage and the future of the EU ETS market. Impact of recent development in the EU ETS on the list of sectors deemed to be exposed to carbon leakage» CE Delft n. ${ }^{\circ}$ 13.7917.18 (abril 2013). 
cierto riesgo de «carbon leakage», es decir a aquellas intensivas en el uso de carbono o con alta exposición al comercio externo, garantizándoles una cobertura del $100 \%$ de sus costes de emisión ${ }^{29}$.

Esta decisión, aunque importante en la consolidación del consenso interno, fue muy criticada tanto por los defensores de una política de cambio climático más coherente. No hay evidencia empírica que las excepciones generalizadas como la utilizada por la UE en 2009 puedan realmente reducir los riesgos de deslocalización ${ }^{30}$. Además, su carácter generalizado y sectorial (cubría aproximadamente un $25 \%$ de todas las industrias incluidas en el sistema de derechos de emisión) era injusto e ineficiente ya que dentro de cada sector podía haber industrias realmente vulnerables junto con otras con capacidad para absorber el sobrecoste o invertir para innovar y reducir las emisiones mediante tecnologías más verdes.

En definitiva, como en toda negociación la diplomacia climática interna ha tenido que realizar ciertas concesiones para poder alcanzar una voz única a nivel internacional. En la materia que estamos estudiando ésta ha estado ligada a la asignación de los derechos de emisión para las actividades con riesgo de pérdida de competitividad. Si bien las reformas introducidas a partir de 2014 intentan evitar las asignaciones de derechos sin coste alguno, lo cierto es que probablemente se mantengan algunas excepciones especialmente para las industrias intensivas en uso de energía.

El problema de la competitividad puede resurgir con fuerza si los acuerdos de Paris finalmente no conducen a planes nacionales compatibles. Es muy probable que los Estados intenten usar la flexibilidad del nuevo marco de Paris para proteger bien a su industria exportadora, bien para impedir la deslocalización de su producción para consumo local. De ser así no sería de extrañar que se creen nuevas excepciones en el esquema europeo y así, aunque con diferentes formas, en parte se haga propio algunos argumentos del discurso americano que acepta tomar medidas contra el cambio climático en la medida que no afecte el núcleo duro de su industria. De hecho los partidos euroescépticos tienen una visión muy fría de la política climática de la UE y de ganar fuerza puede que impon-

29 Decisión de la Comisión 2010/2/UE, de 24 de diciembre de 2009, por la que se determina, de conformidad con la Directiva 2003/87/CE del Parlamento Europeo y del Consejo, una lista de los sectores y subsectores que se consideran expuestos a un riesgo significativo de fuga de carbono [notificada con el número C(2009) 10251] DO L 1 de 5.1.2010, p. $10 / 18$.

${ }^{30}$ Ralf Martin, Mirabelle Muuls, Laure B. de Preux y Ulrich J. Wagner «Industry compensation under relocation risk: A firm-level analysis of the EU emissions trading scheme» The American Economic Review 104, n. ${ }^{\circ} 8$ (2014): 2482-2508. 
gan reformas que posicionen a la UE mucho más cerca de Estados Unidos de lo que pareciera a simple vista.

\section{La evolución del liderazgo europeo}

En la literatura especializada prácticamente no se discute el liderazgo europeo en materia de cambio climático ${ }^{31}$. No es poca cosa, ya que los liderazgos en materia internacional son difíciles de establecer y más aún de mantener. Si bien la historia nos brinda ejemplos de liderazgos morales lo cierto es que normalmente éstos van acompañados de una sólida posición bien esa militar bien sea económica. En lo sustancial la UE cuenta, al menos por el momento, con la ventaja de ser una potencia comercial, aunque en declive. Por ello no es de extrañar que sea dicha tendencia la que marcó la evolución del liderazgo europeo en materia de cambio climático y obligó a mutar de un liderazgo duro a uno más flexible. Lo interesante es que en el camino la diplomacia europea encontró un rol que hasta ahora nunca había ejercido, el de mediador entre las dos superpotencias (China y Estados Unidos) lo que puede deparar unas experiencias muy interesantes para el futuro.

En este apartado hablaremos de «liderazgos» en plural porque, como ha demostrado Charles Parker, Christer Karlsson y Mattias Hjerpe, en materia de cambio climático habría una «fragmentación del liderazgo» ya que no hay ningún actor que supere el $50 \%$ de apoyo ${ }^{32}$. En la actualidad habría más bien tres o cuatro actores principales (tradicionalmente UE, Estados Unidos y China, y más recientemente India) que van variando su influencia según

31 Karin Bäckstrand y Ole Elgström «The EU's Role in Climate Change Negotiations: From Leader to "Leadiator"», Journal of European Public Policy 20, n. ${ }^{\circ} 10$ (2013): 13691386. Michael Grubb y Joyeeta Gupta «Climate Change, Leadership and the EU», en Climante Change and European Leadership, ed. por Michael Grubb y Joyeeta Gupta (Dordrecht: Kluwer, 2010), 3-14. Andrew Jordan et al. Climate Change Policy in the European Union; Confronting the Dilemmas of Mitigation and Adaptation? (Cambridge: Cambridge University press, 2010). Sebastian Oberthür y Claire Roche Kelly «EU Leadership in International Climate Policy: Achievements and Challenges» The International Spectator 43, n. ${ }^{\circ} 2$ (2008): 35-50. Rüdiger Wurzel y James Connelly «Introduction» en The European Union as a Leader in International Climate Change Politics, ed. por Rüdiger Wurzel y James Connelly (Londres: Routledge, 2011): 3-20. Farhana Yamin «The role of the EU in climate negotiations» en Climante Change and European Leadership, ed. por Michael Grubb y Joyeeta Gupta (Dordrecht: Kluwer, 2000), 47-66. Joyeeta Gupta y Lasse Ringius «The EU's climate leadership: Reconciling ambition and reality» International Environmental Agreements: Politics, Law and Economics 1/2 (2001): 281-299.

32 Parker, Karlsson y Hjerpe (2017). 
sus estrategias y las circunstancias. El liderazgo puede seguir cuatro modos principales: estructural, ideológico, direccional e instrumental ${ }^{33}$. La UE ha utilizado los cuatro modos, aunque no siempre al mismo tiempo y con variaciones en su intensidad a lo largo del tiempo.

Finalmente aclarar que el liderazgo europeo es fruto de una aspiración propia $^{34}$ y no siempre se corresponde con un reconocimiento externo. Según una reciente encuesta más allá del mundo occidental, es decir Europa y Norteamérica, el liderazgo europeo en materia de cambio climático es débil en América Latina y Oceanía, y más bien reducido en África y Asia, lo que nos obligará a matizar ciertas generalizaciones ${ }^{35}$.

\section{De la Convención sobre el Cambio Climático al Protocolo de Kioto}

En el período que va desde el inicio de las negociaciones de la Convención sobre Cambio Climático en 1991 hasta la firma del protocolo de Kioto (1997) la UE mantuvo un marcado liderazgo direccional. Este incluyó propuestas concretas y muy ambiciosas como la reducción en un $8 \%$ las emisiones de $\mathrm{CO}_{2}$ para los países industrializadas, iniciativa rechazada en la convención y finalmente aprobadas en el Protocolo de Kioto, que se han tratado de imponer a otros países con la esperanza de que éstos siguieran el «ejemplo» europeo. Pese al fracaso relativo de Kioto, en particular a partir de la salida de los Estados Unidos en 2001, la UE mantuvo esta estrategia, profundizando su modelo interno con reducciones más profundas en el Consejo Europeo de marzo de 2007 (reducción del 20\%

33 Charles F. Parker, Christer Karlsson y Mattias Hjerpe « Climate Change Leaders and Followers: Leadership Recognition and Selection in the UNFCCC Negotiations» International Relations 29, n. 4 (2015): 434-454.

${ }^{34}$ Por ejemplo, en el Consejo de la UE de 9 de marzo de 2007, cuando se debatía el tratado llamado a suceder al Protocolo de Kioto, la UE se auto asignó el rol de liderar una acción global contra el cambio climático hasta 2020 y más allá. Consejo de la Unión Europea «Conclusiones de la Presidencia», Bruselas, 9 de marzo de 2007. En 2012 el presidente Barroso afirmaba que el mundo necesita que la UE lidere la lucha contra el cambio climático. Comisión Europea «Estado de la Unión - 2012», Ref. SPEECH/12/596 (2012). Más recientemente el Comisario para Cambio Climático y Energía afirmaba que el acuerdo de París fue un gran éxito de la diplomacia europea. Miguel Arias Cañete «How Will the Paris Agreement Impact EU Climate and Energy Policies?» Ref. Speech/16/264 (2016).

${ }^{35}$ Según una encuesta realizada por Parker, Karlsson y Hjerpe en París el liderazgo europeo sería percibido como tal por el $17 \%$ de los consultados africanos, $22 \%$ asiáticos, $69 \%$ europeos, $51 \%$ norteamericanos, $37 \%$ sudamericanos y $38 \%$ de Oceanía. Parker, Karlsson y Hjerpe (2017): 245. 
respecto a valores de 1990), y ha ido más allá en la estrategia 2030 con un objetivo de bajar incluso un $40 \%$ las emisiones respecto a la misma fecha base $^{36}$.

Este liderazgo ha sido calificado de «direccional», «liderazgo a través del ejemplo», «diplomacia, persuasión y argumentación» y se vincula a la idea de la UE como «civilian power» ${ }^{37}$. Sin menoscabar las bondades teóricas del modelo lo cierto es que en materia de cambio climático la estrategia ha tenido resultados dispares. Es verdad que ha logrado poner el problema en la agenda internacional, alcanzando una colaboración muy importante con organismos dependientes de Naciones Unidas y la comunidad científica internacional (liderazgo ideológico). Sin embargo, en cuando a la «exportabilidad del modelo» ha tenido poco impacto fuera de las fronteras de la UE. El principio de responsabilidades compartidas pero diferenciadas dejó fuera a los países en desarrollo. Estados Unidos no llegó a ratificar el Protocolo y Canadá lo abandonó en la cumbre de Durban en 2011. Prácticamente sólo Australia, Nueva Zelanda y Japón se unieron al modelo europeo junto con el éxito de la incorporación tardía de Rusia, aunque con efectos dudosos a largo plazo.

Paradójicamente el protocolo de Kioto ha tenido mayores efectos internos que externos. Antes de 1997 la política europea en materia de cambio climático era más bien limitada. Hasta 1993 no hubo un mecanismo para el monitoreo de las emisiones de gases de efecto invernadero. La propuesta de la Comisión para crear un impuesto europeo combinando $\mathrm{CO}_{2}$ y energía no logró el apoyo necesario en el Consejo. Una propuesta sobre reducción de emisiones fue fuertemente rechazada, en particular, por presiones de los fabricantes de coches. La mayor parte de las reducciones de emisiones durante este período se debieron más bien a políticas nacionales, como el reemplazo del carbón por gas en Reino Unido y Alemania, que a iniciativas propiamente europeas. De hecho en 1994 las emisiones eran aún mayores que en $1990^{38}$.

La política en materia de cambio climático no fue realmente europea sino hasta la Directiva 2003/87/CE que posibilitó la creación del Programa Europeo de Derechos de Emisiones. El sistema se fue completando poco a poco con un conjunto de directivas y reglamentos ligados a diferentes aspectos de la política energética y medioambiental con incidencia en

${ }^{36}$ Para un análisis del contenido de la política europea en materia de cambio climático desde una perspectiva española ver Rosa Giles Carnero Cambio climático, energía y derecho internacional: perspctivas de futuro (Cizur Menor: Aranzadi, 2012).

37 Oberthür y Kelly (2008).

38 Ibid.. 
la reducción de emisiones ${ }^{39}$ que, sin embargo, se desarrollaron en pocos años después de la puesta en marcha del Protocolo de Kioto a través de los acuerdos de Marrakech de 2001.

Cabe la duda, por lo tanto, si la UE impulsaba en Kioto más que un modelo una retórica ${ }^{40}$, o un modelo teórico que, una vez aprobado el acuerdo, se convirtió en una profecía autocumplida. Hay autores que afirman que cuando la UE defendió en Kioto la reducción del $15 \%$ «no tenía ni idea de cómo alcanzarlo» ${ }^{41}$. Lo que sí queda claro es que el relato se convirtió en realidad y a partir de entonces la UE ha pasado a ser un líder indiscutible en las negociaciones internacionales en materia de cambio climático.

\section{El fracaso de Copenhague}

El protocolo de Kioto expiraba en 2012, y en vistas a renegociar su continuación se convocó una convención en Copenhague en 2009. La UE siguió básicamente la misma estrategia anterior, tratando de imponer un modelo (reducción vinculante de emisiones) ampliando esta vez el número

39 Directiva 2001/77/CE del Parlamento Europeo y del Consejo, de 27 de septiembre de 2001, relativa a la promoción de la electricidad generada a partir de fuentes de energía renovables en el mercado interior de la electricidad. DO L 283 de 27.10.2001, p. 33/40; Directiva 2002/91/CE del Parlamento Europeo y del Consejo, de 16 de diciembre de 2002, relativa a la eficiencia energética de los edificios. DO L 1 de 4.1.2003, p. 65/71; Directiva 2003/30/CE del Parlamento Europeo y del Consejo, de 8 de mayo de 2003, relativa al fomento del uso de biocarburantes u otros combustibles renovables en el transporte. DO L 123 de 17.5.2003, p. 42/46; Directiva 2004/101/CE del Parlamento Europeo y del Consejo, de 27 de octubre de 2004, por la que se modifica la Directiva 2003/87/CE, por la que se establece un régimen para el comercio de derechos de emisión de gases de efecto invernadero en la Comunidad con respecto a los mecanismos de proyectos del Protocolo de Kioto. DO L 338 de 13.11.2004, p. 18/23; Directiva 2004/8/CE del Parlamento Europeo y del Consejo, de 11 de febrero de 2004, relativa al fomento de la cogeneración sobre la base de la demanda de calor útil en el mercado interior de la energía y por la que se modifica la Directiva 92/42/CEE. DO L 52 de 21.2.2004, p. 50/60; Directiva 2006/32/CE del Parlamento Europeo y del Consejo, de 5 de abril de 2006, sobre la eficiencia del uso final de la energía y los servicios energéticos y por la que se deroga la Directiva 93/76/CEE del Consejo. DO L 114 de 27.4.2006, p. 64/85; Reglamento (CE) n o 842/2006 del Parlamento Europeo y del Consejo, de 17 de mayo de 2006 sobre determinados gases fluorados de efecto invernadero. DO L 161 de 14.6.2006, p. 1/11; y la Directiva 2006/40/CE del Parlamento Europeo y del Consejo, de 17 de mayo de 2006, relativa a las emisiones procedentes de sistemas de aire acondicionado en vehículos de motor y por la que se modifica la Directiva 70/156/CEE del Consejo. DO L 161 de 14.6.2006, p. 12/18.

40 Yamin (2000).

${ }^{41}$ David G. Victor The collapse of the Kyoto Protocol and the struggle to slow global warming. (Princeton: Princeton University Press, 2001): 115. 
de países obligados a algunos países emergentes (particularmente China) y, por supuesto, al principal emisor (Estados Unidos).

Si bien la UE contaba como aval una sólida política climática europea (poco antes el Consejo había acordado incluso un objetivo de reducción de emisiones del 85/90\% para 2050) erró en el modo persuadir a sus socios (liderazgo instrumental).

Tanto en Copenhague (donde se alcanzó un simple acuerdo político que las partes no ratificaron) como en Cancún (donde se incorporaron estas aspiraciones en la Convención sobre Cambio Climático) no se pudieron dar respuesta a los diferentes intereses en juego ya presentes en su día en Kioto pero que habían estado latentes hasta entonces. Probablemente la UE pecó de paternalista al intentar simplemente imponer «su» modelo ${ }^{42}$ (liderazgo estructural) cuando los principales actores, incluido los emergentes, si bien compartían en parte la visión general de la lucha con el cambio climático no estaban dispuestos a adoptar un esquema que pudiese poner en peligro su crecimiento. La crisis de 2008 había minado las economías y la confianza sobre los modelos de desarrollo y los países emergentes no estaban dispuestos, simplemente, a integrarse a un plan prediseñado. Tampoco la alianza transatlántica fue un punto de apoyo sólido. De hecho más que confrontación hubo desinterés. Las negociaciones en muchos casos corrían a través de una suerte de alianza Estados Unidos-China que movió el centro de gravedad de las relaciones internacionales al Pacífico ${ }^{43}$. A continuación trataremos de describir las razones del desastre.

Una parte del fracaso se debió a las carencias negociadoras de la UE como consecuencia de su propia estructura interna de toma de decisiones ${ }^{44}$. Una de las mayores críticas a este respecto se debió a la descoordinación interna de la propia Comisión y de ésta con los Estados miembros que, dado que estamos ante un acuerdo mixto, formaron parte directamente de la negociación. Si bien son conocidas las negociaciones paralelas llevadas a cabo

${ }^{42}$ Hablando del paquete clima-energía aprobado poco antes, en 2008, la Comisión Europea pretendía que fuese «a clear signal to our international partners about our determination to address climate change and should convince them to follow our example». Comisión Europea, «Climate Change: Commission Welcomes Final Adoption of Europe's Climate and Energy Package» Ref. IP/08/1998 (2008).

${ }^{43}$ Christian Egenhofer y Anton Georgiev «The Copenhagen accord-A first stab at deciphering the implications for the EU». CEPS commentary (25 diciembre 2009) acceso el 30 diciembre 2016, https://www .ceps.eu/publications/copenhagen-accord-first-stab-decipheringimplications-eu.

${ }^{44}$ John Vogler. «The European Union as an actor in international environmental politics» Environmental Politics 8, n. 3 (2009): 24-48. Louise van Schaik y Christian Egenhofer «Reform of the EU institutions: Implications for the EU's performance in climate negotiations» CEPS policy brief 40 (1 de septiembre 2003), acceso el 30 diciembre 2016 https://www.ceps. eu/publications/reform-eu-institutions-implications-eus-performance-climate-negotiations. 
en Kioto, donde los ministros de la UE llegaron a una posición común sobre el Mecanismo de Desarrollo Limpio cuando el negociador europeo ya había presentado su propuesta y el acuerdo estaba cerrado ${ }^{45}$, en Copenhague las descoordinaciones y la necesidad de pasar varias veces por diferentes filtros ralentizaron mucho la capacidad negociadora de la UE que se veía desbordada y no llegaba a tiempo para dar respuesta a los problemas planteados por las otras partes contratantes ${ }^{46}$.

Sin embargo, la principal causa del fracaso fue lo desproporcionado de las aspiraciones europeas a los ojos de otros socios, en particular de los Estados Unidos. De haberse aceptado la propuesta europea, los Estados Unidos deberían haberse comprometido a una reducción de emisiones del 30\% cuando la UE sólo necesitaba reducir entre un 3 y un 9\%, Japón un 12\%, Australia un $17 \%$ y Canadá un $29 \%{ }^{47}$. El sacrificio que se estaba pidiendo a los socios era políticamente inviable. La UE estaba actuando más en su propio interés que llevando adelante un liderazgo de beneficios compartidos o win-win como se suele decir ${ }^{48}$. También erró la UE al centrar los esfuerzos negociadores en los países industrializados y no consolidar acuerdos en intereses compartidos con los países en desarrollo, en particular con el G77 y China. No se trataba simplemente de que éstos siguieran el modelo europeo (liderazgo estructural) para lo cual se necesita una fuerte desproporción de poder en favor de la UE que, a todas luces carecía; sino de lograr crear un modelo común mutuamente beneficioso para ambos (liderazgo direccional) y, con el crédito del apoyo de un número importante de Estados, poder convencer a los Estados Unidos y a otros socios desarrollados. Como señala Stavros Afionis ${ }^{49}$ el acercamiento llegó tarde y aisló aún más la posición europea. Como consecuencia el liderazgo de la UE cayó en picado. En el estudio de Parker, Kralsson y Hjerpe entre Poznac y Copenague la percepción del liderazgo europeo a los ojos de terceros se redujo del 62 al $48 \%$. Desde entonces, no hizo más que descender ${ }^{50}$.

45 Michael Grubb y Farhana Yamin «Climatic collapse at The Hague: What happened, why, and where do we go from here? » International Affairs 77 n. ${ }^{\circ} 2$ (2001): 261276, 274.

46 Stavros Afionis «The European Union as a negotiator in the international climate change regime» International environmental agreements: politics, law and economics 11, n. ${ }^{\circ} 4$ (2011): 341-360.

47 Kathryn Harrison, Lisa McIntosh Sundstrom «The comparative politics of climate change» Global Environmental Politics 7, n. ${ }^{\circ} 4$ (2007): 1-18.

${ }_{48}$ Sobre las razones «egoistas» del liderazgo europeo en materia de cambio climático, ver Tora Skodvin and Steinar Andresen «Leadership revisited» Global Environmental Politics 6, n. $^{\circ} 3$ (2006): 13-27.

49 Afionis (2011).

50 Parker, Karlsson y Hjerpe (2017): 247. 


\section{El renacer de la UE como «líder-mediador»: de Durban a París}

El fracaso de Copenhague fue aleccionador y el liderazgo europeo en la materia ya no volvería a ser el mismo. En 2011, en Durban, se sentaron las bases para el cambio. La UE ya no llevaría simplemente un modelo (liderazgo ideológico y direccional), inútil sin una posición de hard power que la sustente (liderazgo estructural) sino que comenzó a tener una política pro-activa para la creación de coaliciones y alianzas (liderazgo instrumental), en primer lugar, con los países emergentes, en segundo término con los menos desarrollados y, finalmente con los socios comerciales más cercanos. Lejos de plantear unos objetivos fijos como en Copenhague la UE, y más concretamente su recientemente creado Servicio Europeo de Acción Exterior, implementó un plan de acción diplomático específico en materia de cambio climático. Dicho plan mejoró mucho la coordinación interna generando sinergias con los servicios diplomáticos de los Estados miembros, como por ejemplo las declaraciones conjuntas de Brasil y Alemania o Francia y China. Cuando dichas alianzas estuvieron consolidadas se exploraron visiones comunes con las políticas nacionales, en particular con los Estados Unidos, para buscar puntos en común y ámbitos de acuerdo ${ }^{51}$. El resultado fue que cuando se llegó a Paris la UE había conseguido conformar una amplia coalición que terminó arrastrando a los socios más duros a la mesa de negociación ${ }^{52}$.

¿Cuál ha sido el secreto del éxito? El primero fue el cambio de estrategia de acción. La UE abandonó el liderazgo clásico (estructural), probablemente alejado de su capacidad real de acción como «actor global» y adoptó una menos ambiciosa pero probablemente más efectiva mezcla de líder con mediador. Los primeros elementos del cambio ya fueron visibles en Durban ${ }^{53}$ y consistieron en asumir que la UE es una potencia «media» en un mundo caracterizado por la multipolaridad con dos grandes actores como lo son China y los Estados Unidos. En este contexto la UE buscó tender puentes entre las dos potencias y los demás países con intereses divergentes. Durante los años que van desde Durban hasta Paris la UE fue el actor más activo en la remisión de propuestas concretas para responder

51 Para un desarrollo detallado de estos pasos previos ver Sebastian Oberthür y Lisanne Groen «The European Union and the Paris Agreement: leader, mediator, or bystander?» Wiley Interdisciplinary Reviews: Climate Change 8 n. ${ }^{\circ} 1$ (2017) doi: 10.1002/wcc.445.

52 Wolfgang Obergassel et al. Phoenix from the ashes - An analysis of the Paris Agreement to the United Nations Framework Convention on Climate Change. Wuppertal Institute for Climate, Environment and Energy (2016), acceso 30 diciembre 2016, http://wupperinst. org/fa/redaktion/downloads/publications/Paris_Results.pdf.

53 Bäckstrand y Elgström (2013). 
a inquietudes variadas planteadas en diferentes foros sobre cambio climático y energía ${ }^{54}$. Cuando la cita se acercaba, no se esperó a la reunión, sino que se preparó el camino con la creación de alianzas primero entre países Norte/Sur (Dialogo de Cartagena por una Acción Progresiva, liderada respectivamente por Reino Unido y Australia) y luego con una gran alianza multilateral llamada «Coalición por una Gran Ambición» comandada por las islas Marshall y financiada por la UE. Si bien eran alianzas con un contenido más bien etéreo dieron a Francia el oxígeno suficiente como para crear una base sobre la cual iniciar los puntos en común durante la convención. Es interesante destacar que la UE, y a diferencia de la era postKioto, no tuvo problemas en ceder el protagonismo dejando en muchos casos a algunos Estados miembros (Alemania, Francia, Reino Unido) e incluso países en principio poco relevantes como las islas Marshall, estar en el foco de atención.

El segundo gran cambio es en la moderación significativa de las aspiraciones, lo que obligó a Estados Unidos y a China a sentarse a negociar. Las posiciones eran las siguientes: La UE pretendía un acuerdo top-down vinculante que requería drásticas reducciones de emisión y un sistema de control externo transparente; los EEUU quería impedir a toda costa el carácter vinculante y era partidario de un sistema más flexible, botton-up, pero compartía con la UE la necesidad de tener un control externo transparente; finalmente China era favorable, al igual que la UE, del carácter vinculante aunque no estaba dispuesto a aceptar una fiscalización externa (transparente) del cumplimiento del plan de reducciones.$^{55} \mathrm{El} \mathrm{G77}$, particularmente los países en desarrollo liderados por India, centraban su interés en la financiación de los planes de reducción.

Respecto a este último elemento, la convención los Estados miembros de la UE comprometieron el cincuenta por ciento de la necesidad financiera del propuesto Fondo Verde para el Clima, haciendo que éste sea una realidad palpable y así ganaron el apoyo de un número significativo de países potencialmente beneficiarios. La flexibilidad europea llegó incluso a la forma del acuerdo, acordándose una fórmula jurídica que permitiese a los Estados Unidos poder firmarlo sin necesitar posteriormente la ratificación del Senado ${ }^{56}$.

En el acuerdo final todos perdieron y ganaron algo. La UE y China no consiguieron que el acuerdo sea vinculante en cuanto a sus objetivos. Par-

54 Oberthür y Groen (2017).

55 Radoslav S. Dimitrov «The Paris Agreement on Climate Change: Behind Closed Doors» Global Environmental Politics 16, n. ${ }^{\circ} 3$ (2016): 1-11, 3.

56 David A. Wirth «Cracking the American Climate Negotiators' Hidden Code: United States Law and the Paris Agreement » Climate Law 6, n. ${ }^{\circ}$ 1-2 (2016): 152-170. 
ticularmente la UE no logró que se fijara un objetivo concreto de reducción, sí en cuanto a la obligación de crear planes nacionales de reducción, cediendo así en parte ante los Estados Unidos. Estos y la UE impusieron a China transparencia en el seguimiento de planes nacionales. El G77 obtuvo financiación, pero perdió la batalla por mantener la diferenciación entre países desarrollados y en desarrollo, y en la creación de instituciones estables, con lo cual se diluye la posibilidad de compensar responsabilidades históricas.

No se puede negar, sin embargo, que la UE contó con elementos externos que funcionaron a su favor. En primer lugar, el presidente Obama, ya finalizando su segundo mandato, estaba especialmente interesado en sumar un legado ambiental a su administración por lo que la delegación americana se encontraba especialmente dispuesta a negociar. En segundo término, y a diferencia de Copenhague, el país anfitrión era una potencia histórica en materia diplomática. Como veremos en el apartado siguiente la presidencia francesa actuó en muchos casos por iniciativa propia de forma separada de la delegación europea asumiendo muchos esfuerzos negociadores por su cuenta y cubriendo lagunas y descoordinaciones ${ }^{57}$. Finalmente China, que se había mostrado inflexible en Copenhague, estaba inmerso en un proceso de transformación económica que la haría virar, antes pronto que tarde, a un modelo de crecimiento más sostenible. Una prueba del cambio fue que poco antes del acuerdo China llegó a consensos bilaterales con la UE en materia cooperación para la creación de un mercado de derechos de emisión y un entendimiento sobre la necesidad de llegar a acuerdos vinculantes en París ${ }^{58}$; lo que contrasta con la sintonía que hubo entre China y los Estados Unidos en Copenhague ${ }^{59}$.

Sea por unas razones o por otras lo cierto es que el acuerdo fue calificado como «histórico» ${ }^{60}$ y seguramente sentará las bases de las negociaciones futuras al menos para las siguientes dos décadas.

${ }^{57}$ Lili Fuhr, Liane Schalatek y Hans Verolme «COP 21 and the Paris Agreement: A Force Awakened» Chinese Journal of Urban and Environmental Studies 4, n. ${ }^{\circ} 01$ (2016): http://dx.doi.org/10.1142/S2345748116500093

58 EU-China Joint Statement on Climate Change, 29 de junio de 2015, acceso el 30 diciembre 2016: http://www.consilium.europa.eu/en/meetings/internationalsummit/2015/06/150629-EU-China-Joint-Statement-on-Climate-Change/

${ }_{59}$ Charles F. Parker, Christer Karlsson y Mattias Hjerpe «Fragmented Climate Change Leadership: Making Sense of the Ambiguous Outcome of COP-15» Environmental Politics 21. n. ${ }^{\circ} 2$ (2012): 268-286.

60 Como calificaron varias delegaciones en la sesión de clausura de la Conferencia de París: «A tremendous collective achievment» (EU), «a marvelous act» (China), «a tremendous victory for the planet» (EEUU), «new chapter of hope in the lives of 7 billion people on the planet» (India). Citado por Dimitrov (2016): 1. 


\section{EI liderazgo europeo más allá de las instituciones de la UE: el rol jugado por la presidencia francesa en los acuerdos de París}

Una de las mayores paradojas del éxito de París es que la UE ha retrocedido como líder global en materia climática a los ojos de otros países. Según Parker, Karlsson y Hjerpe en París la UE habría retrocedido al $41 \%$ (frente al $48 \%$ de Copenhague) siendo los dos actores mejor valorados China (54\%) y los Estados Unidos (59\%) respectivamente ${ }^{61}$. Una explicación es que los terceros países valoran más el cambio de actitud, por parte de China y Estados Unidos, que las razones de dicho cambio, como pueden ser la labor de «líder-mediador» de la UE. Otra razón, apuntada en este caso por los autores del estudio, es que los ocho puntos de retroceso corresponden aproximadamente a la aportación que se le otorga a Francia como contribución para alcanzar el acuerdo final ${ }^{62}$.

Siempre se ha reconocido la importancia del país anfitrión en las negociaciones en materia de cambio climático ${ }^{63}$. Pero en el caso de Paris la presidencia francesa llevó esta influencia a un grado antes nunca visto. La labor del presidente Laurent Fabius y su equipo fue magistral, utilizando hasta el extremo, pero de forma fina y sutil, todos los instrumentos diplomáticos disponibles, incluido un sofisticado sistema de negociación donde el secreto y la política de hechos consumados estuvo a la orden del día. Las negociaciones se llevaban a cabo en grupos reducidos, donde no era posible grabar o siquiera tomar notas. Solo la presidencia guardaba registro de las posiciones de cada uno y, un acuerdo de las partes en un punto, no necesariamente garantizaba su incorporación en el acuerdo final ya que esto dependía de los contrapesos de otras negociaciones paralelas. Esto hacía que el texto final sólo era conocido en el último minuto, forzando una votación global y a libro cerrado ${ }^{64}$. Está claro que tales técnicas pueden ser tachadas de contrarias a la transparencia, pero como el resultado final fue satisfactorio para todas las partes, al final el haber alcanzado un acuerdo legitimó su utilización.

¿Puede considerarse el éxito de la presidencia francesa parte de los méritos de la diplomacia europea? En parte si y en parte no. Si bien Francia asumió la responsabilidad personal de llevar las negociaciones a buen puerto está claro que actuó en coordinación y bajo el impulso de la diplo-

61 Parker, Karlsson y Hjerpe (2017): 245.

62 Ibid., 249.

${ }^{63}$ Siwon Park, «The Power of Presidency in UN Climate Change Negotiations: Comparison Between Denmark and Mexico», International Environmental Agreements: Politics, Law and Economics 16, n. .6 (2016): 781-795.

${ }^{64}$ Dimitrov (2016) : 6/7. 
macia de la UE. No debemos olvidar que la competencia exterior de la UE, también en cambio climático, es compartida con los Estados miembros por lo que toda acción del Servicio Europeo de Acción Exterior y de la Comisión se consulta con los ministerios de asuntos exteriores nacionales. La conclusión más probable es que ambas, la diplomacia de la UE y la francesa, actuaron coordinadamente siendo parte de una diplomacia más general, a la que podemos llamar europea a secas. Esto no debilita la posición de la Unión, sino que la fortalece ya que suma a sus objetivos en materia de cambio climático la experiencia de uno de los servicios diplomáticos nacionales más potentes en términos históricos.

\section{Conclusiones}

Resulta complicado sacar unas conclusiones en relación a la contribución de la UE a la lucha contra el cambio climático en clave de diplomacia medioambiental. Es cierto que la UE ha ejercido y sigue ejerciendo un rol particularmente importante en las negociaciones aunque, como tratamos de demostrar a lo largo de estas páginas, existen muchos matices que en algunos casos ensombrecen y en otros resaltan la acción europea. No pretendemos en estas conclusiones repetir las principales ideas ya expuestas aunque sí intentaremos rescatar algunas enseñanzas que puedan servir para comprender los futuros pasos tanto de la diplomacia en materia de cambio climático como del papel que merece la UE a nivel global.

En primer lugar, hay que decir que la UE no eligió ser el líder en materia de cambio climático sino fue presionada por las circunstancias. Ante el peligro de caer en la irrelevancia internacional la UE se aferró a un papel que le dejaba libre los Estados Unidos e intentó mantenerlo a toda costa. Lo curioso es que en ese juego de intercambio de roles creyó que podía ejercer el mismo papel que el hard power y cayó primero en el aislamiento (Kioto) y luego en la indiferencia (Copenhague).

El cambio de estrategia de Durban y luego de París es positivo, pero no tanto por la flexibilidad que ahora demuestra Europa para aceptar, si se me permite la comparación, una gobernanza climática a «diferentes velocidades», sino más bien por su rol activo de mediador. Es en este juego de triangulación norte-sur y occidente-oriente donde la UE tiene un papel que jugar como potencia media. Sin embargo, tal como ocurre dentro de la Unión, el papel de mediador le exige tomar la iniciativa si se puede antes o al menos inmediatamente después de que surjan los problemas, proponiendo soluciones, tal como hizo los meses previos a Paris. Esto genera un claro coste en tiempo y esfuerzo que la maquinaria diplomática europea sólo se puede permitir en un ámbito relativamente acotado como es el cambio climático. 
Además, en Paris la diplomacia europea se sirvió de la aceitada maquinaria del Quai d'Orsay, perdiendo peso y visibilidad a los ojos de terceros, pero ganando en resultados.

En cuanto al fondo, la duda que surge es si verdaderamente el acuerdo de Paris crea un entramado de planes nacionales de reducción de emisiones, y la política climática muta verdaderamente hacia una nueva gobernanza de economía sostenible (ahora también llamada economía circular ${ }^{65}$ ) ¿Podrá realmente la UE vencer sus contradicciones internas y seguir manteniendo el papel de líder mediador multilateral? Las contradicciones todavía latentes en el sistema europeo que protege a sus industrias altamente contaminantes si existen riesgos de deslocalización o incluso permitir abusos en los controles de emisiones de industrias con poder de presión como la de coches nos demuestran que en algunas cosas no nos diferenciamos tanto de los americanos. Es más, el reforzamiento de los partidos ultra nacionalistas quedará reflejado, como se vislumbra en algunas de las medidas de la nueva administración Trump, con lo que la cohesión interna será aún más difícil que hasta ahora.

Las tensiones en materia de competitividad seguramente serán un foco de conflicto importante y si la UE quiere mantener el liderazgo tendrá que centrar su atención en resolverlas proponiendo soluciones equitativas, paulatinas y sostenibles. Al fin y a cabo se trata de administrar un problema económico, un campo en el que la UE tiene una gran experiencia negociadora.

\section{Bibliografía}

Afionis, Stavros «The European Union as a negotiator in the international climate change regime» International environmental agreements: politics, law and economics 11, n. 4 (2011): 341-360.

Arias Cañete, Miguel «How Will the Paris Agreement Impact EU Climate and Energy Policies?» Ref. Speech/16/264 (2016).

Bäckstrand, Karin y Ole Elgström «The EU's Role in Climate Change Negotiations: From Leader to "Leadiator"», Journal of European Public Policy 20, n. ${ }^{\circ} 10$ (2013): 1369-1386.

Chasek, Pamela «US policy in the UN environmental arena: powerful laggard or constructive leader?», International Environmental Agreements: Politics, Law and Economics 7, n. 4 (2007): 363-387.

Comisión Europea «Climate Change: Commission Welcomes Final Adoption of Europe's Climate and Energy Package» Ref. IP/08/1998 (2008).

65 Beatriz Pérez de las Heras «La gestión eficiente de recursos en la unión europea: alcance e impacto de la normativa europea para una economía más sostenible y circular» $R e$ vista de Derecho Comunitario Europeo 55 (2016): 781-818. 
Comisión Europea «Estado de la Unión - 2012», Ref. SPEECH/12/596 (2012).

Consejo de la Unión Europea «Conclusiones de la Presidencia», Bruselas, 9 de marzo de 2007.

Corti Varela, Justo, «EU Emission Trading System, competitiveness and taxes on the energy sector» en State Aids, Taxation and the Energy Sector, ed. por Marta Villar Ezcurra (Cizur Menor: Thomson Reuters, 2017), 323-336.

De Bruyn, Sander, Dagmar Nelissen y Marnix Koopman «Carbon leakage and the future of the EU ETS market. Impact of recent development in the EU ETS on the list of sectors deemed to be exposed to carbon leakage» CE Delft n. 13.7917 .18 (abril 2013).

Dimitrov, Radoslav S. «The Paris Agreement on Climate Change: Behind Closed Doors» Global Environmental Politics 16, n. 3 (2016): 1-11.

Egenhofer, Christian y Anton Georgiev «The Copenhagen accord-A first stab at deciphering the implications for the EU». CEPS commentary (25 diciembre 2009) acceso el 30 diciembre 2016, https://www.ceps.eu/publications/ copenhagen-accord-first-stab-deciphering-implications-eu.

Frémion, Yves, Histoire de la révolution écologiste (Paris: Hoëbeke, 2007).

Fuhr, Lili, Liane Schalatek y Hans Verolme «COP 21 and the Paris Agreement: A Force Awakened» Chinese Journal of Urban and Environmental Studies 4, n. ${ }^{\circ} 01$ (2016): http://dx.doi.org/10.1142/S2345748116500093

Giles Carnero, Rosa, Cambio climático, energía y derecho internacional: perspctivas de futuro (Cizur Menor: Aranzadi, 2012).

Groen, Lisanne y Arne Niemann, «The European Union at the Copenhagen Climate Negotiations: A Case of Contested EU Actorness and Effectiveness» International Relations 27, n. 3 (2013): 308-324.

Grubb, Michael y Farhana Yamin «Climatic collapse at The Hague: What happened, why, and where do we go from here? » International Affairs 77 n. 2 (2001): 261-276.

Grubb, Michael y Joyeeta Gupta «Climate Change, Leadership and the EU», en Climante Change and European Leadership, ed. por Michael Grubb y Joyeeta Gupta (Dordrecht: Kluwer, 2010), 3-14.

Gupta, Joyeeta y Lasse Ringius «The EU's climate leadership: Reconciling ambition and reality» International Environmental Agreements: Politics, Law and Economics 1/2 (2001): 281-299.

Harrison, Kathryn, Lisa McIntosh Sundstrom «The comparative politics of climate change» Global Environmental Politics 7, n. 4 (2007): 1-18.

Jordan, Andrew, Dave Huitema, Harro van Asselt, Timothy Rayner y Frans Berkhout Climate Change Policy in the European Union; Confronting the Dilemmas of Mitigation and Adaptation? (Cambridge: Cambridge University press, 2010).

Kelemen, R. Daniel y David Vogel, «Trading places: the role of the United States and the European Union in international environmental politics», Comparative Political Studies 43, n. ${ }^{\circ} 4$ (2010): 427-456.

Kelemen, R. Daniel y Tim Knievel, «The United States, the European Union, and international environmental law: the domestic dimensions of green diplomacy!», International Journal of Constitutional Law 13, n. 4 (2015): 945-965. 
Marcu. Andrei, Christian Egenhofer, Susanna Roth y Wijnand Stoefs «Carbon Leakage: An overview» CEPS Special Report (2013), acceso 30 de diciembre 2016, www.ceps.be/book/carbon-leakage-overview.

Martin, Ralf, Laure de Preux y Ulrich Wagner «The impacts of a carbon tax on manufacturing: Evidence from microdata» Journal of Public Economics 117, n. ${ }^{\circ} 1$ (2014): 1-14.

Martin, Ralf, Mirabelle Muuls, Laure B. de Preux y Ulrich J. Wagner «Industry compensation under relocation risk: A firm-level analysis of the EU emissions trading scheme» The American Economic Review 104, n. 8 (2014): 24822508.

Obergassel, Wolfgang, Christof Arens, Lukas Hermwille, Nico Kreibich, Florian Mersmann, Hermann E. Ott, y Hanna Wang-Helmreich Phoenix from the ashes - An analysis of the Paris Agreement to the United Nations Framework Convention on Climate Change. Wuppertal Institute for Climate, Environment and Energy (2016), acceso 30 diciembre 2016, http://wupperinst.org/fa/ redaktion/downloads/publications/Paris_Results.pdf.

Oberthür, Sebastian y Claire Roche Kelly «EU Leadership in International Climate Policy: Achievements and Challenges» The International Spectator: Italian Journal of International Affairs 43, n. 2 (2008): 35-50.

Oberthür, Sebastian y Lisanne Groen «The European Union and the Paris Agreement: leader, mediator, or bystander?» Wiley Interdisciplinary Reviews: Climate Change 8 n. 1 (2017) doi: 10.1002/wcc.445.

Oreskes, Naomi, y Erik M. Conway, Merchants of doubt: How a handful of scientists obscured the truth on issues from tobacco smoke to global warming (Nueva York: Bloomsbury Publishing, 2011): 169-215.

Park, Siwon «The Power of Presidency in UN Climate Change Negotiations: Comparison Between Denmark and Mexico», International Environmental Agreements: Politics, Law and Economics 16, n. ${ }^{\circ} 6$ (2016): 781-795.

Parker, Charles F., Christer Karlsson y Mattias Hjerpe «Climate Change Leaders and Followers: Leadership Recognition and Selection in the UNFCCC Negotiations» International Relations 29, n. ${ }^{\circ} 4$ (2015): 434-454.

Parker, Charles F., Christer Karlsson y Mattias Hjerpe «Assessing the European Union's global climate change leadership: from Copenhagen to the Paris Agreement» Journal of European Integration 39, n. 2 (2017): 239-252, DOI: 10.1080/07036337.2016.1275608.

Parker, Charles F., Christer Karlsson y Mattias Hjerpe «Fragmented Climate Change Leadership: Making Sense of the Ambiguous Outcome of COP-15» Environmental Politics 21. n. ${ }^{\circ} 2$ (2012): 268-286.

Pérez de las Heras, Beatriz «La gestión eficiente de recursos en la unión europea: alcance e impacto de la normativa europea para una economía más sostenible y circular» Revista de Derecho Comunitario Europeo 55 (2016): 781-818.

Skodvin, Tora and Steinar Andresen «Leadership revisited» Global Environmental Politics 6, n. 3 (2006): 13-27.

Turner, James Morton, «The Specter of Environmentalism: Wilderness, Environmental Politics, and the Evolution of the New Right», The Journal of American History 96, n. 1 (2009): 123-148. 
van Schaik, Louise y Christian Egenhofer «Reform of the EU institutions: Implications for the EU's performance in climate negotiations» CEPS policy brief 40 (1 de septiembre 2003), acceso el 30 diciembre 2016 https://www .ceps. eu/publications/reform-eu-institutions-implications-eus-performance-climatenegotiations.

Victor, David G., The collapse of the Kyoto Protocol and the struggle to slow global warming. (Princeton: Princeton University Press, 2001).

Vig, Norman J. y Michael Gebert Faure (eds.) Green Giants? Environmental Policies of the United States and the European Union (Cambridge: MIT Press, 2004).

Vogel, David, The politics of precaution: regulating health, safety, and environmental risks in Europe and the United States (Princeton: Princeton University Press, 2012): 235-248.

Vogler, John «The European Union as an actor in international environmental politics» Environmental Politics 8, n. ${ }^{\circ} 3$ (2009): 24-48.

Wirth, David A. «Cracking the American Climate Negotiators' Hidden Code: United States Law and the Paris Agreement» Climate Law 6, n. ${ }^{\circ}$ 1-2 (2016): 152-170.

Wurzel, Rüdiger K.W., Duncan Liefferink y James Connelly «Introduction. European Union climate leadership» en The European Union in International Climate Change Politics: Still Taking a Lead?, ed. por Rudiger K.W. Wurzel, James Connelly, y Duncan Liefferink (Abingdon: Routledge, 2017): 1-17.

Wurzel, Rüdiger y James Connelly «Introduction» en The European Union as a Leader in International Climate Change Politics, ed. por Rüdiger Wurzel y James Connelly (Londres: Routledge, 2011): 3-20.

Yamin, Farhana «The role of the EU in climate negotiations» en Climante Change and European Leadership, ed. por Michael Grubb y Joyeeta Gupta (Dordrecht: Kluwer, 2000), 47-66.

\section{Sobre el autor}

Justo Corti Varela, profesor adjunto de Derecho Internacional Público y Unión Europea en la Universidad CEU San Pablo. Acreditado a TU por la ANECA. Un sexenio de investigación reconocido por la CNEAI. Investigador senior del Instituto de Estudios Europeos. Doctor en derecho por la Universidad Complutense de Madrid, Master en UE por la misma universidad. Profesor visitante de la Université de Nanterre (Paris X), Investigador Post-doct del CNRS (Paris), Investigador visitante del UCL (Londres) y de la Scoula Superiore Sant'Anna (Pisa). Sus áreas de investigación son el Derecho Medioambiental Internacional, el Derecho Económico Internacional, la Unión Europea, la regulación internacional de las nuevas tecnologías. Es miembro del consejo de redacción de la RDCE y de Ius et Scientia. 


\begin{abstract}
About the author
Justo Corti Varela is adjunct professor of Public International Law and European Union at CEU San Pablo University. Evaluated positively as Senior Lecturer by ANECA. He has a period of research recognized by CNEAI. Senior researcher at the Institute for European Studies. PhD in law and LLM in EU by Complutense University. Visiting professor of Université de Nanterre (Paris X), Post-doctoral researcher at CNRS (Paris), and visiting scholar at UCL (London) and at Scoula Superiore Sant'Anna (Pisa). His areas of research are International Environmental Law, International Economic Law, European Union, and the regulation of new technologies. He is member of the board of the RDCE and Ius et Scientia.
\end{abstract}




\section{Derechos de autor}

Los derechos de autor (para la distribución, comunicación pública, reproducción e inclusión en bases de datos de indexación y repositorios institucionales) de esta publicación (Cuadernos Europeos de Deusto, CED) pertenecen a la editorial Universidad de Deusto. El acceso al contenido digital de cualquier número de Cuadernos Europeos de Deusto es gratuito inmediatamente después de su publicación. Los trabajos podrán leerse, descargarse, copiar y difundir en cualquier medio sin fines comerciales y según lo previsto por la ley; sin la previa autorización de la Editorial (Universidad de Deusto) o el autor. Así mismo, los trabajos editados en CED pueden ser publicados con posterioridad en otros medios o revistas, siempre que el autor indique con claridad y en la primera nota a pie de página que el trabajo se publicó por primera vez en $C E D$, con indicación del número, año, páginas y DOI (si procede). Cualquier otro uso de su contenido en cualquier medio o formato, ahora conocido o desarrollado en el futuro, requiere el permiso previo por escrito del titular de los derechos de autor.

\section{Copyright}

Copyright (for distribution, public communication, reproduction and inclusion in indexation databases and institutional repositories) of this publication (Cuadernos Europeos de Deusto, CED) belongs to the publisher University of Deusto. Access to the digital content of any Issue of Cuadernos Europeos de Deusto is free upon its publication. The content can be read, downloaded, copied, and distributed freely in any medium only for non-commercial purposes and in accordance with any applicable copyright legislation, without prior permission from the copyright holder (University of Deusto) or the author. Thus, the content of $C E D$ can be subsequently published in other media or journals, as long as the author clearly indicates in the first footnote that the work was published in $C E D$ for the first time, indicating the Issue number, year, pages, and DOI (if applicable). Any other use of its content in any medium or format, now known or developed in the future, requires prior written permission of the copyright holder. 